\title{
CONVERGENCE AND STABILITY ANALYSIS OF AN EXPLICIT FINITE DIFFERENCE METHOD FOR 2-DIMENSIONAL REACTION-DIFFUSION EQUATIONS
}

\author{
NIAN LI ${ }^{1}$, JOSEPH STEINER ${ }^{1}$ and SHIMIN TANG ${ }^{2}$
}

(Received 28 July 1992; revised 5 February 1993)

\begin{abstract}
The convergence and stability analysis of a simple explicit finite difference method is studied in this paper. Conditional convergence and stability theorems for this method are given. We have also proved that this scheme is stable in a much stronger sense.
\end{abstract}

\section{Introduction}

We consider the following 2-dimensional reaction-diffusion equation

$$
\begin{gathered}
\frac{\partial u}{\partial t}=\lambda_{1} \frac{\partial^{2} u}{\partial x^{2}}+\lambda_{2} \frac{\partial^{2} u}{\partial y^{2}}+f(u), \\
0<t \leq T, \quad(x, y) \in \Omega, \\
u(x, y, 0)=u^{0}(x, y), \\
u(x, y, t)=0, \quad(x, y) \in \partial \Omega,
\end{gathered}
$$

where

$$
\Omega=\{(x, y)|| x|<a,| y \mid<b\}
$$

and

$$
f(u) \in C^{1}[0,1]
$$

satisfying

$$
f(0)=f(1)=0 .
$$

The above equation states that the rate of increase of a quantity $u(x, y, t)$ depends on the effects of diffusion and reaction. This interaction between diffusion and reaction

\footnotetext{
${ }^{1}$ Mathematics Department, Swinburne University of Technology, Hawthom 3122, Australia.

${ }^{2}$ Department of Mechanics, Peking University, Beijing, China.

(C) Australian Mathematical Society, 1994, Serial-fee code 0334-2700/94
} 
occurs in many natural phenomena such as the propagation of a virile mutant in an infinitely long habitat, the evolution of a neutron population in a nuclear reactor, the spreading of flames in a burning forest or in a chamber of combustion (see [14], [2] and [17]). Recently the above equation has been used as a model equation to study the phenomena of bifurcation, self-organization and chaos for complex nonlinear dynamic systems [7]. For this reason the reaction-diffusion equation constitutes an important area of mathematical research. Aronson and Weinberger first studied this multidimensional nonlinear equation and obtained many significant theoretical results [2].

Various numerical methods for solving the equation such as the pseudo- spectral method and the alternating group explicit iterative method [4] [3], have been applied, however both are quite complicated and the former approach always causes instability. Several finite element methods have been employed [1] [8] with excellent accuracy and stability, but they take quite a long CPU time. In order to obtain an efficient and stable method, Tang et al. [15] recently had no other recourse but to reinvoke the finite difference method. This method, although regarded by some as unsophisticated, works quite fast since it does not need the inverses of large matrices. Their numerical experiments show that the scheme is numerically stable, without any theoretical justification. It is the object of this paper to provide a rigorous mathematical justification for using FDM to solve the 2-D reaction-diffusion equation, and at the same time derive simple conditions on the space-time grid which will guarantee its convergence and stability to a practical solver of this equation at a glance.

The concepts of consistency, convergence and stability have provided the necessary basis for wide research of efficient difference schemes for problems of mathematical physics. A lot of significant work regarding the convergence and stability of FDM has been done for linear problems. There is a well known Lax's equivalence theorem that is for a consistent finite difference scheme, stability is equivalent to convergence [13]. This theorem is only valid for linear equations. So, for our problem we have to investigate both the convergence and the stability of the scheme separately.

We give a conditional convergence theorem in Section 3 and a conditional stability theorem in Section 4. The concept of stability of a difference scheme with respect to rounding errors, for given initial conditions, was first described by von Neumann and Richtmeyer in 1950 [16]. Later on, several slightly different definitions of stability were presented (see [5] pages 475-476). In this paper we use the one presented by Lax and Richtmeyer [13] in which the boundedness of a difference operator is the criterion. The von Neumann condition for stability is applied for the proof of the stability theorem.

Surprisingly, our theorems show that even for this nonlinear problem the conditions for convergence and stability are identical. At the end of Section 4 we give a result that with suitable choice of parameters, the scheme is stable even in a much stronger 
sense.

The above, somewhat restrictive, assumptions about the nonlinear term $f(u)$ are justified because in most applications

$$
f(u)=u^{n}(1-u), \quad n=1,2, \ldots
$$

However, our results are also valid if $f$ is Lipschitz, instead of $C^{1}$.

The stability and convergence of FDM for systems of nonlinear reaction-diffusion equations have been already considered by Hoff [9] in an arbitrary number of space dimensions, on an arbitrary domain, with coefficients general functions of space, time and unknown, for a system of equations, and for implicit as well as the explicit algorithm. However, Hoff's paper is too theoretical and it does not prescribe readily amenable conditions sought by a practical solver of the widely used 2-D reactiondiffusion equation considered here. Moreover his definition of stability is also stronger than the commonly accepted definitions used in our paper, hence more difficult to achieve in practice. Furthermore, our analysis is intentionally restricted to a simple but commonly used explicit scheme which requires much less CPU time than an implicit one.

The necessary description of the difference scheme and notations are given in Section 2.

\section{Scheme and notations}

We divide the $\Omega$ into $m n \Delta x \times \Delta y$ small rectangles, where $\Delta x=2 a / n$ and $\Delta y=2 b / m$. The node points $\left(x_{i}, y_{j}\right)$ in the grids are

$$
\begin{aligned}
& x_{i}=-a+i \Delta x \quad i=0,1, \ldots, n, \\
& y_{j}=-b+j \Delta y \quad j=0,1, \ldots, m .
\end{aligned}
$$

The notations

$$
U_{i, j}^{p}=U\left(x_{i}, y_{j}, t_{p}\right)
$$

and

$$
u_{i, j}^{p}=u\left(x_{i}, y_{j}, t_{p}\right)
$$

are used to denote the approximate and exact solutions respectively at $\left(x_{i}, y_{j}\right)$, at the time $t=t_{p}=p \Delta t$.

The simple explicit difference scheme then can be described by the following equations

$$
\begin{array}{r}
U_{i, j}^{p+1}=U_{i, j}^{p}+\alpha\left(U_{i+1, j}^{p}-2 U_{i, j}^{p}+U_{i-1, j}^{p}\right)+ \\
\beta\left(U_{i, j+1}^{p}-2 U_{i, j}^{p}+U_{i, j-1}^{p}\right)+\Delta t f\left(U_{i, j}^{p}\right)
\end{array}
$$


for $i=1,2, \ldots, n-1$ and $j=1,2, \ldots, m-1$, where

$$
\alpha=\lambda_{1} \Delta t / \Delta x^{2} \quad \text { and } \quad \beta=\lambda_{2} \Delta t / \Delta y^{2} .
$$

In the computation grid in the $x y$-plane, the nodes inside the region are ordered in a natural way - from the top left to the bottom right. The corresponding $U$ values at these nodes at time $p \Delta t$ in the same order form the vector

$$
\begin{aligned}
\vec{U}^{p}= & {\left[U_{1}^{p}, U_{2}^{p}, \ldots, U_{(n-1)(m-1)}^{p}\right]^{T} } \\
\equiv & {\left[U_{1, m-1}^{p}, U_{2, m-1}^{p}, \ldots, U_{n-1, m-1}^{p},\right.} \\
& U_{1, m-2}^{p}, U_{2, m-2}^{p}, \ldots, U_{n-1, m-2}^{p}, \\
& \left.\ldots \ldots, U_{1,1}^{p}, U_{2,1}^{p}, \ldots, U_{n-1,1}^{p}\right]^{T} .
\end{aligned}
$$

Then the scheme can be expressed in the matrix form

$$
\vec{U}^{p+1}=A \vec{U}^{p}+\Delta t \operatorname{diag}\left\{f\left(U_{1}^{p}\right), f\left(U_{2}^{p}\right), \ldots, f\left(U_{(n-1)(m-1)}^{p}\right)\right\},
$$

where

$$
A=\left[\begin{array}{cccccc}
\Gamma & \beta I_{n-1} & 0 & \cdots & 0 & 0 \\
\beta I_{n-1} & \Gamma & \beta I_{n-1} & \cdots & 0 & 0 \\
\vdots & \vdots & \vdots & \ddots & \vdots & \vdots \\
0 & 0 & 0 & \cdots & \Gamma & \beta I_{n-1} \\
0 & 0 & 0 & \cdots & \beta I_{n-1} & \Gamma
\end{array}\right]
$$

is an $(n-1)(m-1) \times(n-1)(m-1)$ matrix,

$$
\Gamma=\left[\begin{array}{cccccc}
1-2 \alpha-2 \beta & \alpha & 0 & \cdots & 0 & 0 \\
\alpha & 1-2 \alpha-2 \beta & \alpha & \cdots & 0 & 0 \\
\vdots & \vdots & \vdots & \ddots & \vdots & \vdots \\
0 & 0 & 0 & \cdots & 1-2 \alpha-2 \beta & \alpha \\
0 & 0 & 0 & \cdots & \alpha & 1-2 \alpha-2 \beta
\end{array}\right]
$$

is an $(n-1) \times(n-1)$ matrix and $I_{n-1}$ is the $(n-1) \times(n-1)$ identity matrix. We use $\rho($.) to denote the spectral radius of a matrix (see [6, page 508]).

\section{A convergence theorem}

The local truncation error of our scheme at $\left(x_{i}, y_{j}, t_{p}\right)$ is

$$
\begin{array}{r}
\tau_{i, j}^{p} \equiv \frac{u_{i, j}^{p+1}-u_{i, j}^{p}}{\Delta t}-\lambda_{1} \frac{u_{i+1, j}^{p}-2 u_{i, j}^{p}+u_{i-1, j}^{p}}{\Delta x^{2}} \\
-\lambda_{2} \frac{u_{i, j+1}^{p}-2 u_{i, j}^{p}+u_{i, j-1}^{p}}{\Delta y^{2}}-f\left(u_{i, j}^{p}\right) .
\end{array}
$$


Considering $u$ is the exact solution of (1) and using Taylor's series expansion, we have

$$
\begin{aligned}
\tau_{i, j}^{p}=(\Delta t / 2) \frac{\partial^{2} u\left(x_{i}, y_{j}, \tilde{t}\right)}{\partial t^{2}}-\left(\Delta x^{2} / 12\right) \lambda_{1} \frac{\partial^{4} u\left(\tilde{x}_{i}, y_{j}, t_{p}\right)}{\partial x^{4}} & \\
& -\left(\Delta y^{2} / 12\right) \lambda_{2} \frac{\partial^{4} u\left(x_{i}, \tilde{y}_{j}, y_{p}\right)}{\partial y^{4}} .
\end{aligned}
$$

We assume that $u_{t}, u_{x x x x}$ and $u_{y y y y}$ are continuous in $\bar{\Omega} \times[0, T]$. So there are constants $K_{1}, K_{2}$ and $K_{3}$ such that

$$
\left|\tau_{i, j}^{p}\right| \leq K_{1} \Delta t+K_{2} \Delta x^{2}+K_{3} \Delta y^{2} \equiv E .
$$

Rearranging the terms of (4), we have

$$
\begin{aligned}
u_{i, j}^{p+1}=u_{i, j}^{p}+\alpha\left(u_{i+1, j}^{p}-2 u_{i, j}^{p}+u_{i-1, j}^{p}\right)+ \\
\beta\left(u_{i, j+1}^{p}-2 u_{i, j}^{p}+u_{i, j-1}^{p}\right)+f\left(u_{i, j}^{p}\right) \Delta t+\tau_{i, j}^{p} \Delta t .
\end{aligned}
$$

Let

$$
e_{i, j}^{p}=u_{i, j}^{p}-U_{i, j}^{p}
$$

We subtract (2) from (6) and assume $1-2 \alpha-2 \beta \geq 0$.

After taking magnitudes of both sides of the new equation, the following inequality is then obtained

$$
\begin{aligned}
\left|e_{i, j}^{p+1}\right| \leq(1-2 \alpha-2 \beta)\left|e_{i, j}^{p}\right|+\alpha\left|e_{i-1, j}^{p}\right|+\alpha\left|e_{i+1, j}^{p}\right| \\
+\beta\left|e_{i, j-1}^{p}\right|+\beta\left|e_{i, j+1}^{p}\right|+K_{4}\left|e_{i, j}^{p}\right| \Delta t+E \Delta t,
\end{aligned}
$$

where $K_{4}$ is the maximum magnitude of $f^{\prime}(u)$. If we let

$$
e^{p}=\max _{1 \leq i \leq n-1,1 \leq j \leq m-1}\left|e_{i, j}^{p}\right|,
$$

then the above inequality becomes

$$
e^{p+1} \leq\left(1+K_{4} \Delta t\right) e^{p}+E \Delta t
$$

showing that

$$
e^{p} \leq\left(1+K_{4} \Delta t\right)^{p} e^{0}+\left[1+\left(1+K_{4} \Delta t\right)+\cdots+\left(1+K_{4} \Delta t\right)^{p-1}\right] E \Delta t .
$$

Since $e^{0}=0$ and $p \Delta t \leq T$, we have

$$
\begin{aligned}
e^{p} & \leq \frac{\left(1+K_{4} \Delta t\right)^{p}-1}{K_{4} \Delta t} E \Delta t \\
& \leq \frac{\left(1+K_{4} \Delta t\right)^{T / \Delta t}-1}{K_{4}} E \\
& \leq \frac{\exp \left(K_{4} T\right)-1}{K_{4}} E \rightarrow 0 \quad \text { as } \Delta x, \Delta y \text { and } \Delta t \rightarrow 0 .
\end{aligned}
$$

Thus we have proved the following theorem . 
THEOREM 1. If the solution of (1) has continuous $u_{t}, u_{x x x x}$ and $u_{y y y y}$ in $\Omega$, then the approximate solution generated by the simple difference scheme (2) converges to the exact one as $\Delta t, \Delta x$ and $\Delta y$ tend to zero, keeping $\alpha+\beta \leq 1 / 2$.

\section{Stability analysis}

Applying the Mean Value Theorem to the difference operator (3) we get

$$
\vec{U}^{p+1}=\left(A+\Delta t \operatorname{diag}\left\{f^{\prime}\left(\tilde{U}_{1}^{p}\right), f^{\prime}\left(\tilde{U}_{2}^{p}\right), \ldots, f^{\prime}\left(\tilde{U}_{(n-1)(m-1)}^{p}\right)\right\}\right) \vec{U}^{p}
$$

or

$$
\vec{U}^{p+1}=A_{p} \vec{U}^{p}
$$

where

$$
\begin{aligned}
A_{p} & =A+\Delta A_{p} \\
& =A+\Delta t \operatorname{diag}\left\{f^{\prime}\left(\tilde{U}_{1}^{p}\right), f^{\prime}\left(\tilde{U}_{2}^{p}\right), \ldots, f^{\prime}\left(\tilde{U}_{(n-1)(m-1)}^{p}\right)\right\} .
\end{aligned}
$$

It is easy to verify that if $1-2 \alpha-2 \beta \geq 0$ then $\|A\|_{\infty}=1$.

So the spectral radius of $A, \rho(A) \leq\|A\|_{\infty}=1$. Since both $A$ and $\Delta A_{p}$ are symmetric and $\rho\left(\Delta A_{p}\right) \leq K_{4} \Delta t$, we have the following inequalities (see [6, page 411])

$$
\rho\left(A_{p}\right)=\rho\left(A+\Delta A_{p}\right) \leq \rho(A)+\rho\left(\Delta A_{p}\right) \leq 1+K_{4} \Delta t .
$$

Therefore the von Neumann necessary condition for stability is satisfied, that is

$$
\rho\left(A_{p}\right) \leq 1+K_{4} \Delta t .
$$

Since $A_{p}$ is symmetric the condition is also sufficient if the matrix 2-norm is used [13]. The following theorem is then proved.

THEOREM 2. The difference scheme (2) is stable if $\alpha+\beta \leq 1 / 2$.

The essence of stability is that there should be a limit to the extent to which any initial error can be amplified in the numerical procedure. It is easy to see that if the scheme satisfies a stronger condition

$$
\rho\left(A_{p}\right) \leq 1
$$

then the initial error will not be amplified at all, in fact it tends to zero if $\rho\left(A_{p}\right)$ is strictly less than one. This kind of stability was first studied by O'Brien, Hyman and Kaplan [12]. The following theorem shows that the simple explicit scheme is also conditionally stable in the sense of B-H-K. 
THEOREM 3. If $\alpha+\beta \leq 1 / 2$ and $\Delta t \leq(1-\rho(A)) / K_{4}$, then $\rho\left(A_{p}\right) \leq 1$.

PROOF OF THEOREM 3. The key point of the proof is to show that $\rho(A)$ is strictly less than 1. Let $k$ and $l$ be two positive integers less than $n$ and $m$ respectively, and $V^{k l}$ an $(n-1)(m-1)$-dimensional vector having

$$
\sin \frac{k i \pi}{n} \sin \frac{l j \pi}{m}
$$

as the $(i+(j-l)(n-1))$-th component for $i=1, \ldots, n-1$ and $j=1, \ldots, m-1$. It is easy to see that the $(i+(j-1)(n-1))$-th component of $A V^{k l}$ is

$$
\begin{aligned}
& \beta \sin \frac{k i \pi}{n} \frac{l(j-1) \pi}{m}+\alpha \sin \frac{k(i-1) \pi}{n} \sin \frac{l j \pi}{m}+(1-2 \alpha-2 \beta) \sin \frac{k i \pi}{n} \sin \frac{l j \pi}{m} \\
&+\alpha \sin \frac{k(i+1) \pi}{n} \sin \frac{l j \pi}{m}+\beta \sin \frac{k i \pi}{n} \sin \frac{l(j+1) \pi}{m}
\end{aligned}
$$

which can be simplified to

$$
\left(1-4 \alpha \sin ^{2} \frac{k \pi}{2 n}-4 \beta \sin ^{2} \frac{l \pi}{2 m}\right) \sin \frac{k i \pi}{n} \sin \frac{l j \pi}{m} .
$$

Thus $A V^{k l}=\mu^{k l} V^{k l}$, showing that $V^{k l}$ is an eigenvector of $A$ with eigenvalue $\mu^{k l}$, where

$$
\mu^{k l}=1-4 \alpha \sin ^{2} \frac{k \pi}{2 n}-4 \beta \sin ^{2} \frac{l \pi}{2 m}
$$

for $1 \leq k \leq n-1$ and $1 \leq l \leq m-1$. Therefore, if $\alpha+\beta \leq 1 / 2$ then

$$
\rho(A)=\mu^{11}=1-4 \alpha \sin ^{2} \frac{\pi}{2 n}-4 \beta \sin ^{2} \frac{\pi}{2 m}<1 .
$$

The rest of the proof is just reapplying the inequality $\rho\left(A_{p}\right) \leq \rho(A)+\rho\left(\Delta A_{p}\right)$ which is used in the proof of Theorem 2.

Thus the proof is completed.

REMARK. We can see if $\rho\left(A_{p}\right)<1$ the numerical solution $\vec{U}^{p}$ will tend to the one of the nonlinear algebraic system

$$
\vec{U}=A \vec{U}+\Delta t \operatorname{diag}\left\{f\left(U_{1}\right), f\left(U_{2}\right), \ldots, f\left(U_{(n-1)(m-1)}\right\}\right.
$$

as $p$ tends to infinity. By the well known Contraction Mapping Theorem, such a nonzero solution is unique. This coincides with the result obtained by Tang et al. [15] that claims that various local initial disturbances will lose their own colourful structure. This means that the steady state solution is independent of the initial $u^{0}$. 


\section{Acknowledgement}

We wish to thank the referees for their constructive comments. The explicit formula for the eigenvalues of $A$ was suggested by one of the referees.

\section{References}

[1] J. Argyris, M. Haase and J. C. Heinrich, "Finite approximation to two-dimensional sin-Gordon equations", Comput. Methods Appl. Mech. Engrg. 86 (1991) 1-26.

[2] D. G. Aronson and H. F. Weinberger, "Multidimensional nonlinear diffusion arising in population genetics", Adv. in Math. 30 (1978) 33-76.

[3] D. J. Evans and M. S. Sahimi, "The alternating group explicit (AGE) iterative method to solve parabolic and hyperbolic partial differential equations", Ann. of Numerical Fluid Mechanics and Heat Transfer 2 (1989) 283-389.

[4] J. Gazdag and J. Canosa, "Numerical solutions of Fisher's equation", J. Appl. Probab. 11 (1974) 445-457.

[5] S. K. Godunov and V. S. Ryabenkii, Difference schemes (Elsevier Science, New York, 1987).

[6] G. H. Golub and C. F. Van Loan, Matrix computations (John Hopkins, Baltimore, 1989).

[7] D. F. Griffiths and A. R. Mitchell, "Stable periodic bifurcations of an explicit discretization of a nonlinear partial differential equation in reaction diffusion", IMA J. Numer. Anal. 8 (1988) 435-454.

[8] R. Grimshaw and S. Tang, "The rotation-modified Kadomtsev-Petviashvili equation: an analytical and numerical study", Stud. Appl. Math. 83 (1990) 223-248.

[9] D. Hoff, "Stability and convergence of finite difference methods for systems of nonlinear reactiondiffusion equations”, SIAM J. Numer. Anal. 15 (6) (1978) 1161-1177.

[10] E. Isaacson and H. B. Keller, Analysis of numerical methods (Wiley, New York, 1966).

[11] G. I. Marchuk, Methods of numerical mathematics (Springer-Verlag, New York, 1975).

[12] G. G. O'Brien, M. A. Hyman and S. Kaplan, "A study of the numerical solution of partial differential equation", J. Math. Phys. 29 (1951) 223.

[13] R. Richtmeyer and K. W. Morton, Difference methods for initial value problems (Wiley, New York, 1967).

[14] S. Tang, S. Qin and R. O. Weber, "Numerical solution of a nonlinear reaction-diffusion equation", Applied mathematics and mechanics, English edition 12 (8) (Aug. 1991) 751-758.

[15] S. Tang, S. Qin and R. O. Weber, "Numerical studies on 2-dimensional reaction-diffusion equations", J. Austral. Math. Soc. Ser. B 35 (1993) 223-243.

[16] J. von Neumann and R. D. Richtmeyer, "A method for the numerical calculation of hydrodynamical shocks", J. Appl. Phys. 21 (1950) 232.

[17] G. B. Yu and A. R. Mitchell, "Analysis of a non-linear difference scheme in reaction-diffusion", Numer. Math. 49 (1986) 511-527. 